\title{
TOFtracker: combination of time-of-flight and high-accuracy bidimensional tracking in a single gaseous detector
}

\section{A. Blanco}

LIP-Laboratório de Instrumentação e Física Experimental de Partículas Dep. de Física, Univ. de Coimbra, 3004-516 Coimbra, Portugal

E-mail: alberto@coimbra.lip.pt

\section{P. Fonte}

LIP-Laboratório de Instrumentação e Física Experimental de Partículas Dep. de Física, Univ. de Coimbra, 3004-516 Coimbra, Portugal ISEC-Instituto Superior de Engenharia de Coimbra

Rua Pedro Nunes - Quinta da Nora, 3030-199 Coimbra, Portugal

E-mail: fonte@coimbra.lip.pt

\section{Lopes}

LIP-Laboratório de Instrumentação e Física Experimental de Partículas Dep. de Física, Univ. de Coimbra, 3004-516 Coimbra, Portugal

E-mail: luisalberto@coimbra.lip.pt

\section{P. Martins}

LIP-Laboratório de Instrumentação e Física Experimental de Partículas Dep. de Física, Univ. de Coimbra, 3004-516 Coimbra, Portugal

E-mail: pmartinsecoimbra. Iip.pt

J. Michel

Institut für Kernphysik, Goethe-Universität

Max-von-Laue-Str. 1, 60438 Frankfurt am Main

E-mail: michelephysik.uni-frankfurt.de

\section{Palka}

Institut für Kernphysik, Goethe-Universität

Max-von-Laue-Str. 1, 60438 Frankfurt am Main

E-mail: M.Palka@gsi.de

\section{Kajetanowicz}

Nowoczesna Elektronika

ul. Bolestwa Prusa 15/10, 30-109 Cracow, Poland

E-mail: mkajetan@ne.com.pl

\footnotetext{
$1 \quad$ Speaker
} 


\title{
G. Korcyl
}

Jagiellonian University

ul. Gotębia 24, 31-007 Cracow, Poland

E-mail: grzegorz.korcyl@gmail.com

\section{Traxler}

GSI Helmholtz Centre for Heavy Ion Research

Planckstraße 1, 64291 Darmstadt, Germany

E-mail: M.Traxleregsi.de

\section{R. Ferreira Marques}

LIP-Laboratório de Instrumentação e Física Experimental de Partículas

Dep. de Física, Univ. de Coimbra, 3004-516 Coimbra, Portugal

E-mail: ruiecoimbra.lip.pt

\begin{abstract}
A 5-gap timing RPC equipped with patterned electrodes coupled to both charge-sensitive and timing circuits yields a time accuracy of $77 \mathrm{ps}$ along with a position accuracy of $38 \mu \mathrm{m}$. These results were obtained by calculating the straight-line fit residuals to the positions provided by a 3-layer telescope made out of identical detectors, detecting almost perpendicular cosmic-ray muons. The device may be useful for particle identification by time-of-flight, where simultaneous measurements of trajectory and time are necessary.
\end{abstract}

XI workshop on Resistive Plate Chambers and Related Detectors (RPC2012)

INFN-Laboratori Nazionali di Frascait, Italy

February 5-10, 2012

\section{Introduction}

Particle identification by the time-of-flight technique depends on the combination of timing and tracking detectors. This is generally performed by detectors specialized for each task. However there may be advantages in performing these measurements by detectors capable of performing both tasks simultaneously $([1,2])$, as exemplified in Fig. 1. For instance, each particle would be measured several times, improving timing accuracy (although on a shorter path) and there is no need for an external initial time detector, which is sometimes problematic.

The capability of Resistive Plate Chambers (RPCs) to deliver a position resolution below $0.1 \mathrm{~mm}$ has been already demonstrated for thin single-gap devices working in digital readout mode and its application to tracking discussed [3]. However this technique cannot be applied to wide-gap or multigap RPCs owing to their comparatively larger thickness, spreading the induced charge profile.

Likewise, the capability of RPCs to deliver time resolutions close to 50 ps has been established for several years, but only in devices with a position resolution of a few mm [4] at most.

In this study we demonstrate for the first time the simultaneous measurement of accurate time and space coordinates in a single gaseous detector, demonstrating as well the application of 
classical charge interpolation readout to obtain the position of charged particles in RPCs to accuracy better than $0.1 \mathrm{~mm}$.

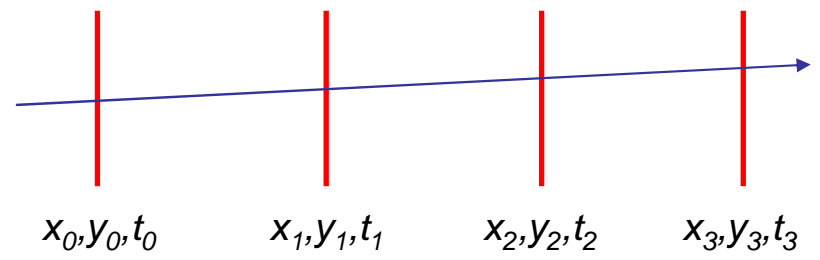

Fig. 1 - A tracking system capable of simultaneously measurement of accurate coordinates and time may have advantages. For instance, each particle would be measured several times, improving timing accuracy (although on a shorter path) and there is no need for an external initial time detector, which is sometimes problematic.

\section{Detector}

The detector had an active area of $8 \times 8 \mathrm{~cm}^{2}$ and was composed by a multigap RPC [5] with five gas gaps of $0.35 \mathrm{~mm}$ width delimited by $0.4 \mathrm{~mm}$ thick soda-lime glass plates. The high voltage is applied to each side of the stack by thin electrodes made of a semiconducting polymer $^{2}$ epoxied to the outer glasses and insulated from the readout electrodes by a kapton foil.

Signals were induced on $4 \mathrm{~mm}$ pitch metallic strips formed on a thin printed circuit board, as depicted in Fig. 2. Each X-strip delivers some of the induced charge to a common PCB track via a $40 \mathrm{pF}$ capacitor and the remainder to a charge amplifier via a choke ${ }^{3}$. The common track is connected to a custom timing amplifier and comparator somewhat similar to [6] via two equal-length cables (see Fig. 3). This arrangement directs the high-frequency signal components towards the timing amplifiers and the low frequencies towards the charge amplifiers. It should be noted that this way it is possible to use the total induced charge for position determination and not just the prompt component.

The Y-strips are arranged in the perpendicular direction and the arrangement is similar to the X-strips, except that now the common track is split in two, each side of the Y-strips connecting to these via $20 \mathrm{pF}$ capacitors. The shielding of the cables leading to the timing amplifiers is connected to these tracks.

Three similar detectors are stacked vertically, spaced by $5 \mathrm{~cm}$, forming a 3-layer telescope (Fig. 3).

The strip signals are fed via the chokes to integrating amplifiers with $10 \mu$ s integration time, digitized by $40 \mathrm{MHz}$ streaming ADCs and digitally filtered in the time domain by a trapezoidal filter (e.g. [7]) with time constants $2 \mu \mathrm{s}, 1 \mu \mathrm{s}, 2 \mu \mathrm{s}$ (leading, top, falling). The particle passing times were recorded by $100 \mathrm{ps}$ bin TDCs. See [8] for further info on the data acquisition system.

\footnotetext{
${ }^{2}$ SEMITRON $®$.

${ }^{3}$ MURATA BLM21BB201SH1.
} 

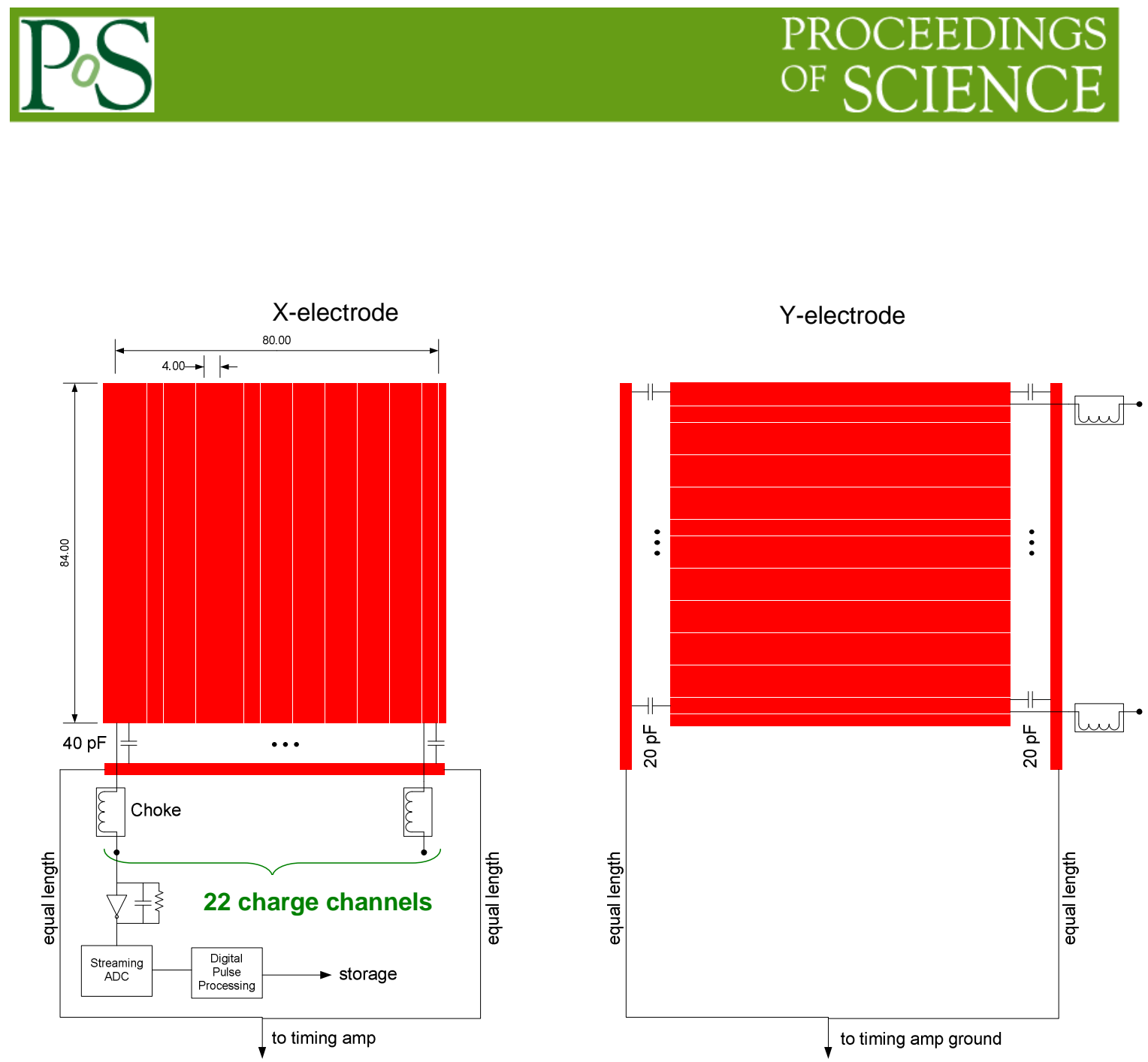

Fig. 2 - Schematic representation of the coordinate-reading electrodes and the readout circuit.

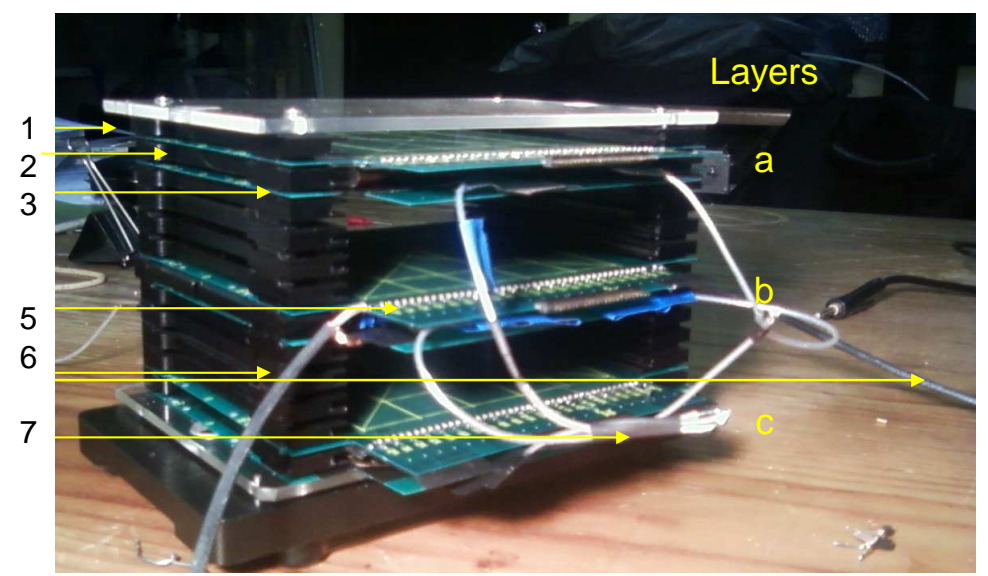

Fig. 3 - View of the telescope. Legend: 1-X strips; 2-RPC; 3-Y strips; 5-signal division network; 6-equal length cables connecting to the timing amplifier; 7-HV connections.

\section{Charge interpolation}

The detector cross section, along with the weighting field (e.g. [9]) map for a $4 \mathrm{~mm}$ pitch strip is represented in Fig. 4. The intensity of the vertical weighting field for this generic strip as a function of the avalanche distance in each gap is shown in Fig. 5 a). The charge sharing 
between several strips can be deduced from these curves by considering the different avalanche-strip distances.

To determine the avalanche position from the induced charge pattern, the following algorithm was used. From the charge in the 3 strips with larger signal, $Q_{-1}, Q_{0}, Q_{1}$, the following quantities were formed: $Q=Q_{-1}+Q_{0}+Q_{1}, q_{-1}=Q_{-1} / Q, q_{0}=Q_{0} / Q, q_{1}=Q_{1} / Q$, $S_{1}=\left(q_{1}-q_{-1}\right) / \sqrt{2}, \quad S_{2}=q_{0} / \sqrt{2}-\left(q_{1}-q_{-1}\right) / 2, \alpha=\arctan \left(S_{2} / S_{1}\right)$. In Fig. 5 b $)$ it can be seen the calculated relation between $\alpha$ and the avalanche position relative to the centre of the strip with largest charge for the five cases (gaps) shown in Fig. 5 a), in steps of $0.1 \mathrm{~mm}$. The position coordinates of each event are calculated by forming $\alpha$ and then inverting the average curve, as shown as well in Fig. 5 b).

The bi-dimensional position distribution of 330k events calculated this way is shown in Fig. 6. The three fishing line spacers are clearly visible in each layer. In layers "a" and "c" some differential non-linearity of unknown origin appears in the $\mathrm{Y}$ coordinate.

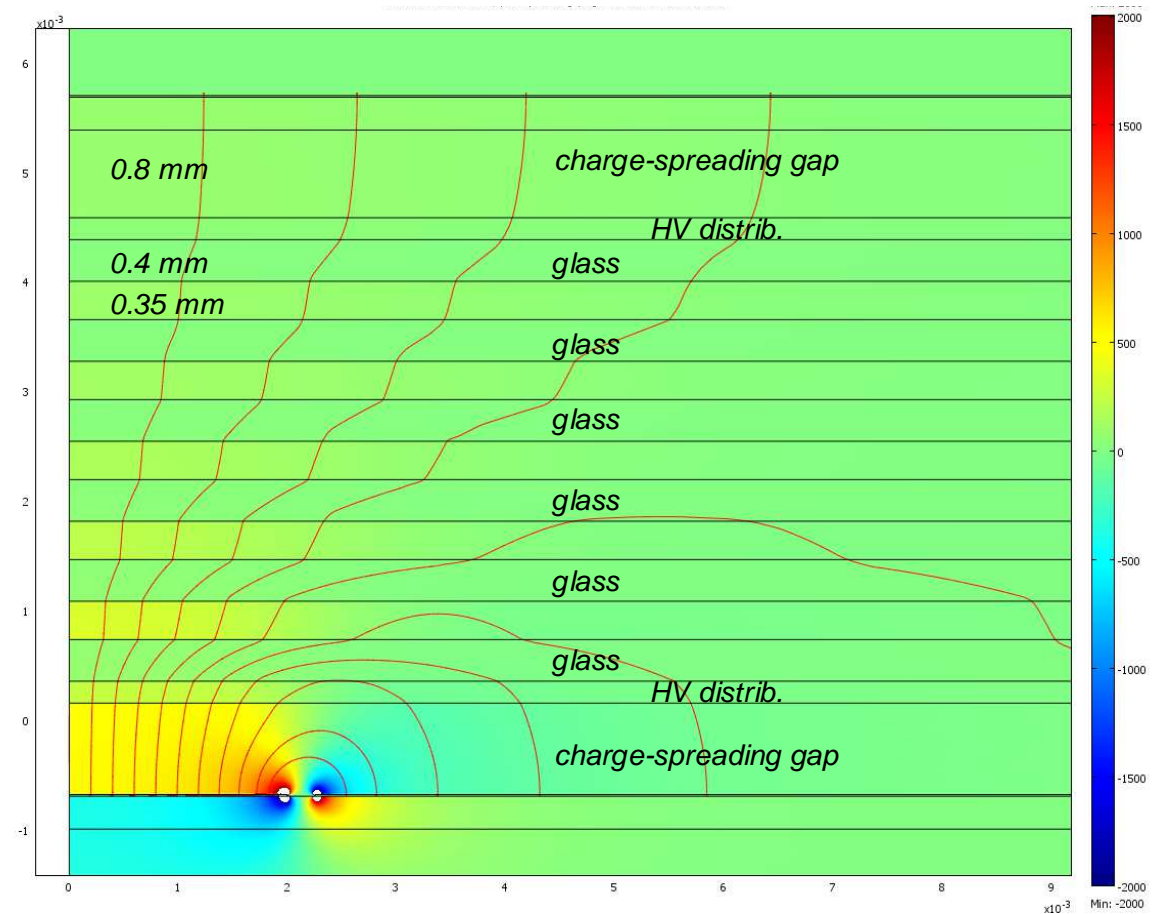

Fig. 4 - Representation of the detector cross-section (in meter) and of the weighting field for a $4 \mathrm{~mm}$ pitch strip. The red lines are the electric field lines and the colour map the intensity of the vertical component of the weighting field (in $\mathrm{V} / \mathrm{m}$ ). The $\mathrm{x}<0$ region was omitted owing to reflection symmetry. 

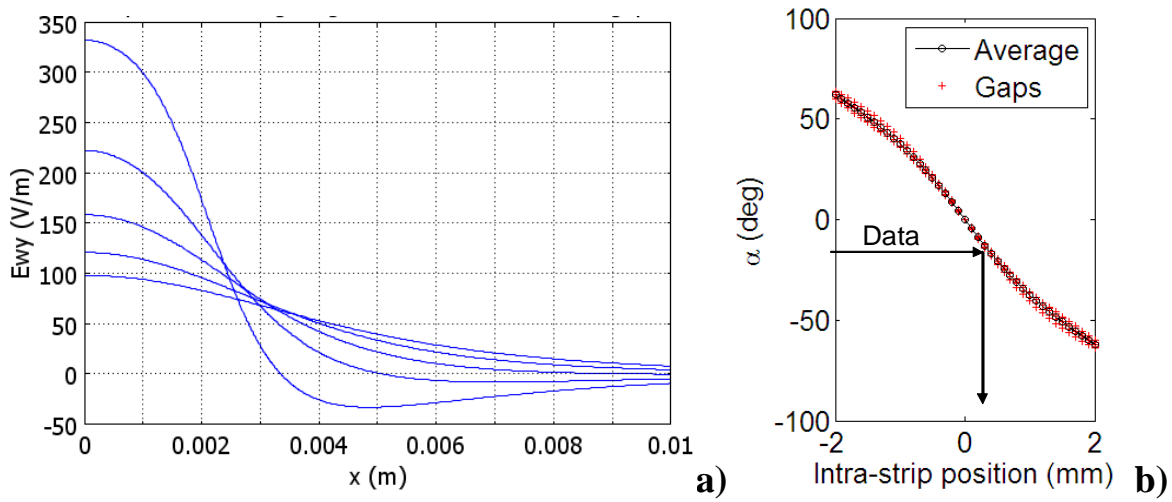

Fig. 5 - a) Intensity of the weighting field in the direction perpendicular to the plates for a generic $4 \mathrm{~mm}$ strip (only right hand side shown) and for each gap (as shown in Fig. 4) as a function of the distance between the avalanche and the centre of the strip. b) Relation between the value $\alpha$ defined in the text and the avalanche position. The average of the curves is also shown. This average is used to estimate the avalanche position from the data by calculating the $\alpha$ value for each coordinate in each event and inverting the function.
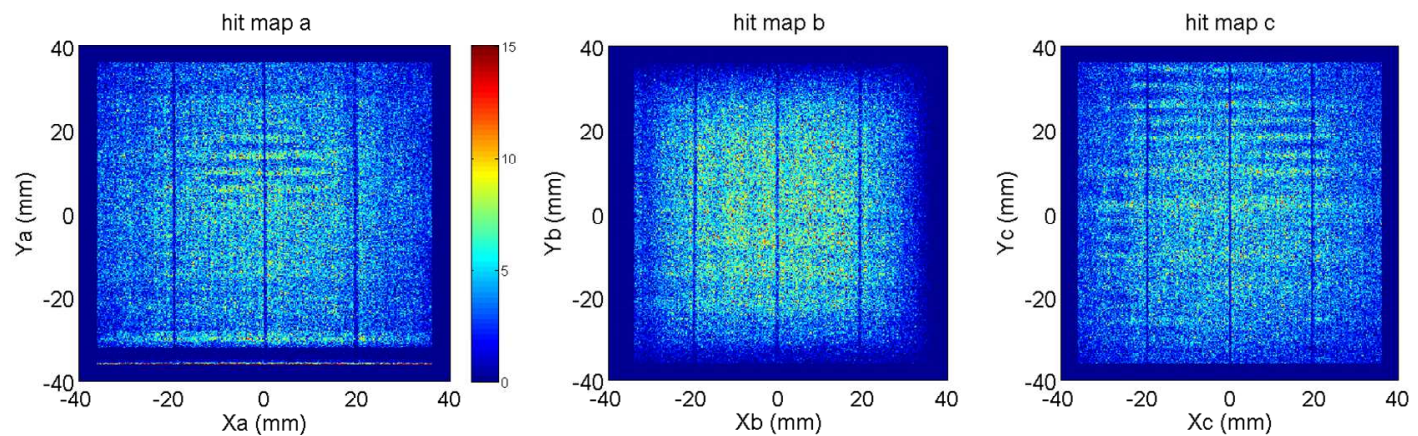

Fig. 6 - Position distribution of the triggered events in each of the 3 layers of the telescope in $0.25 \mathrm{~mm}$ wide bins. The fishing line spacers are clearly visible in all layers. In layers " $\mathrm{a}$ " and "c" some differential non-linearity ("waves") of unknown origin appears in the Y coordinate.

\section{Results}

\subsection{Tracking of cosmic rays}

The 3-layer telescope tracked cosmic rays muons upon a coincidence trigger generated in the outer detectors. Events were retained when the track inclination, $\theta$, relative to the vertical direction was smaller than $\tan (\theta)=1 / 20$. This selects tracks whose $X$ or $Y$ distance between the top and the bottom layers range up to $5 \mathrm{~mm}$, excluding any automatic cancellation of systematic effects that would occur if the tracks were chosen strictly perpendicular to the electrodes, hitting the same region of the same strip in all layers. The events with coordinates within $1 \mathrm{~cm}$ from the edges were excluded because such events show a charge deficit in the outer strips and must be handled via a modified algorithm, to be studied. The effective active area considered here was therefore of $6 \times 6 \mathrm{~cm}^{2}$ for each layer. 

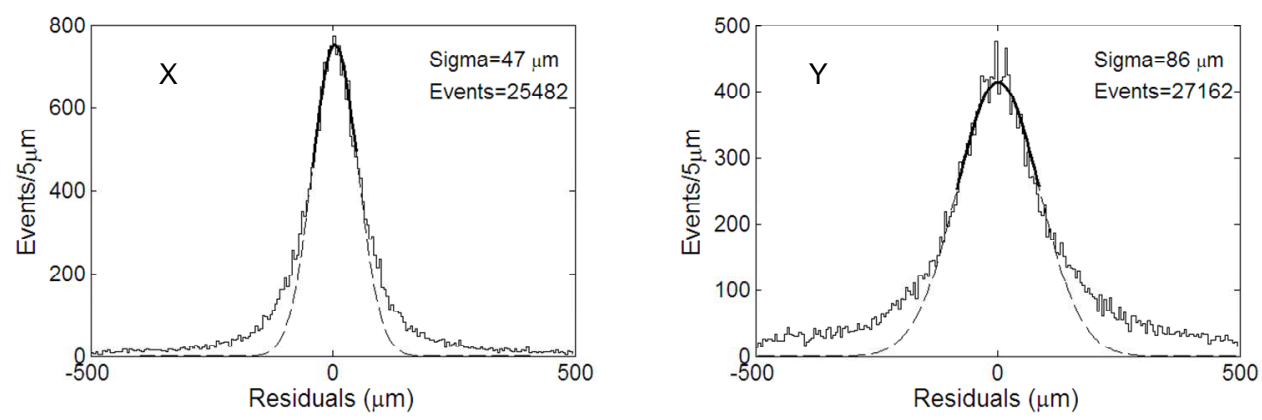

Fig. 7 - Residuals to the straight-line fit to each coordinate of the collected tracks, taking in consideration the full effective area and tracks with moderate, but not negligible, inclination. It is hypothesised that the lower resolution observed in $\mathrm{Y}$ originates from the differential non-linearity evidenced in Fig. 6.

A straight-line fit was performed to the three available positions in each coordinate without any further cuts (therefore taking the whole effective area). In Fig. 7 it can be seen the distribution of the residuals for each coordinate, along with a Gaussian fit performed within $\pm 1 \sigma$. The bulk of the $X$ distribution shows a width of $47 \mu \mathrm{m} \sigma$, while the $Y$ distribution shows a width of $86 \mu \mathrm{m} \sigma$. It is hypothesized that this difference arises from the differential non-linearity evidenced in Fig. 6.

After the procedure described above some systematic errors remain, as the observed fit residuals depend deterministically on the value of corresponding coordinate (Fig. 8). This effect was corrected by using half of the data to determine the offset that should be applied to each $0.2 \mathrm{~mm}$ coordinate interval in each layer to globally minimise the residuals. The offsets were then applied to the other half of the data and the result plotted in Fig. 9. The bulk of the $X$ distribution shows now a width of $38 \mu \mathrm{m} \sigma$, while the $\mathrm{Y}$ distribution shows a width of $71 \mu \mathrm{m} \sigma$. It should be noted that if the data were taken in only a small portion of the detector, not over the entire area, as it would be the case for tests made in a focussed particle beam, such effects would be invisible and the results presented in Fig. 7 and Fig. 9 would be identical.

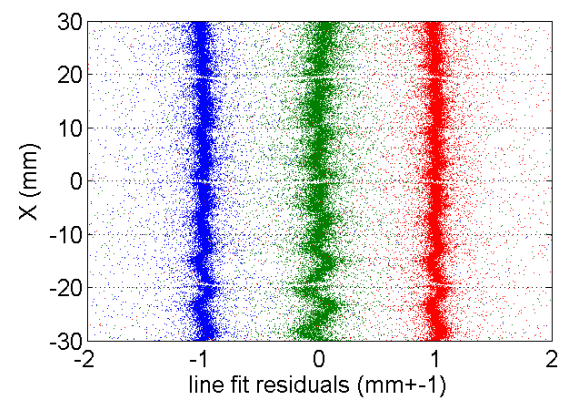

Fig. 8 - Observed systematic dependence of the fit residuals on the corresponding coordinate. For visibility, an offset of $\pm 1 \mathrm{~mm}$ was added to each residual. 

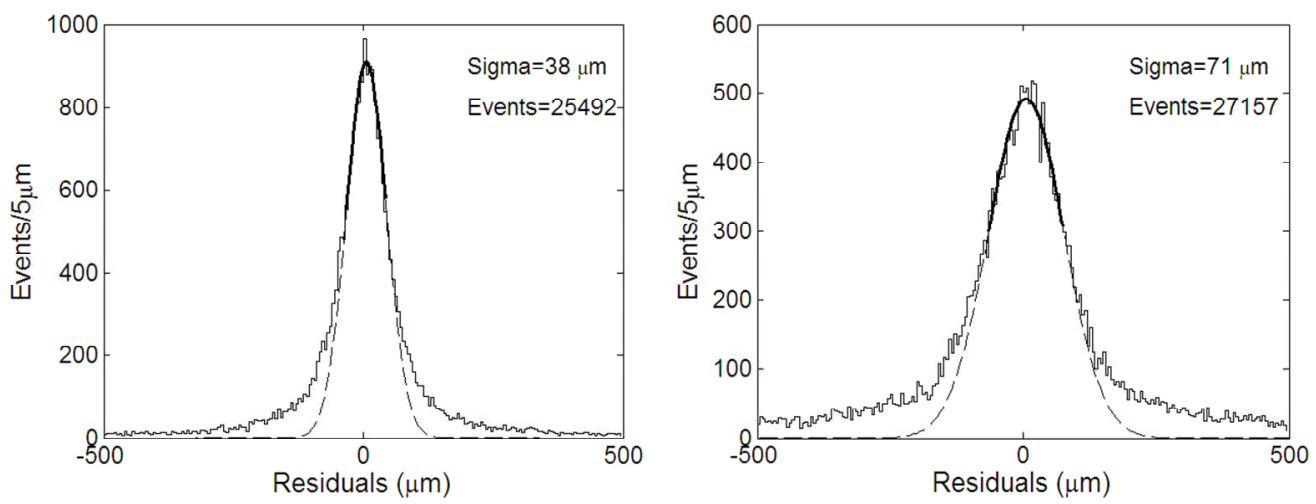

Fig. 9 - Residuals to the straight-line fits to each coordinate of the collected tracks after correction of systematic errors, taking in consideration the full effective area and tracks with moderate, but not negligible, inclination.

\subsection{Time resolution}

The time differences between each layer pair were successively corrected by each of the measured charges in the corresponding layers. It was found that this correction is very much dependent on the location of the tracks. It is hypothesized that this effect is related to a considerable signal dispersion owing to a deficient propagation on crossed strips. To counter this effect the time-charge correction was performed in regions of $1 \mathrm{~cm}^{2}$ and the results concatenated for the final fit, which is shown in Fig. 10. The average single-layer resolution is found to be $77 \mathrm{ps}$ and the telescope resolution (combination of the 3 layers) is $77 / \sqrt{ } 3=44 \mathrm{ps}$.
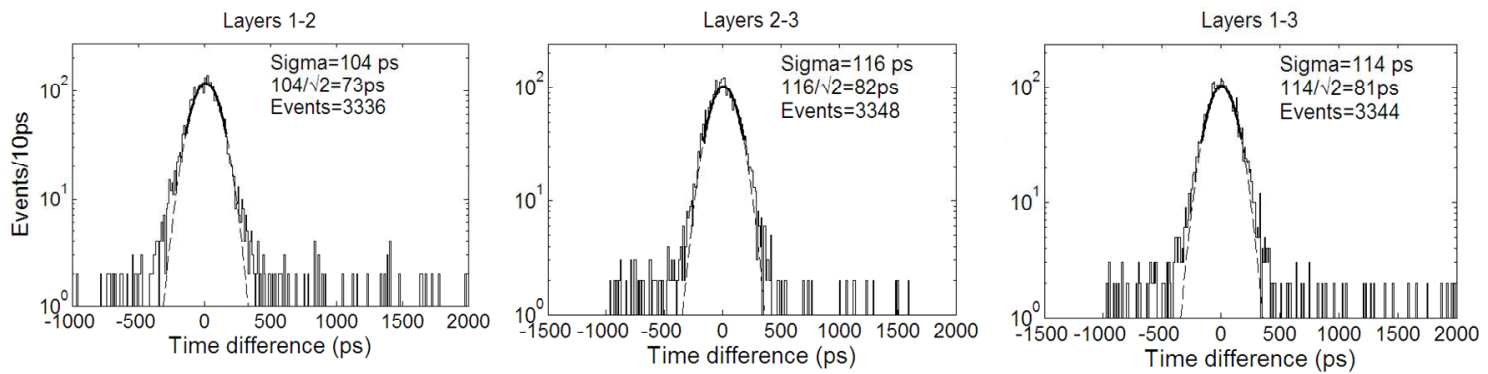

Fig. 10 - Time difference between successive telescope layers after time-charge corrections.

The gaussian fit was performed within $\pm 1.5 \sigma$ of the peak. The average single-layer resolution is $77 \mathrm{ps}$ and the telescope resolution (combination of the 3 layers) is $77 / \sqrt{3}=44 \mathrm{ps}$.

\subsection{Conclusion}

The TOFtracker device, a 5-gap timing RPC readout by charge interpolation between strip electrodes in 2 dimensions, yields a position resolution up to $38 \mu \mathrm{m}$ when forming a 3-layer telescope tracking cosmic-ray muons impinging on a $6 \times 6 \mathrm{~cm}^{2}$ active area. The average single-layer time resolution was 77 ps. 


\section{Acknowledgement}

This work was supported by FCT and FEDER/COMPETE under the contract CERN/FP/116392/2010.

\section{References}

[1] RD51 Proposal, "Position sensitive timing RPCs for time-tagged muon tracking", the RD51 collaboration, 20/8/2008 (http://rd51-public.web.cern.ch/rd51-public/Documents/RD51Proposal_21082008.pdf).

[2] J. A. Garzon et al., Nucl Instrum. and Meth. A 629 (2011) 311.

[3] T. Francke et al., Nucl Instrum. and Meth. A 508 (2003) 83.

[4] A. Blanco et al., Nucl. Instrum. and Meth. A 508 (2003) 70.

[5] E. Cerron-Zeballos et al., Nucl Instrum. and Meth. A 374 (1996) 132.

[6] D. Belver et al., Nuclear Physics B - Proc. Supp. 158 (2006) 47-51.

[7] E. Gatti and P.F.Manfredi, Nuovo Cimento 9 (1986) 75-146.

[8] J. Michel et al., 2011 JINST 6 C12056 (doi: 10.1088/1748-0221/6/12/C12056).

[9] V. Radeka, Ann. Rev. of Nucl. and Part. Sci., 38 (1988) 217-277 (DOI: 10.1146/annurev.ns.38.120188.001245). 